\title{
A critical role for network structure in seizure onset: a computational modeling approach
}

\section{George Petkov ${ }^{1 \dagger}$, Marc Goodfellow ${ }^{1 \dagger}$, Mark P. Richardson ${ }^{2}$ and John R. Terry ${ }^{1}$ *}

${ }^{1}$ College of Engineering, Mathematics and Physical Sciences, University of Exeter, Exeter, UK

2 Institute of Psychiatry, King's College London, London, UK

\section{Edited by:}

David F. Abbott, The Florey Institute of Neuroscience and Mental Health, Australia

\section{Reviewed by:}

Andreas Schulze-Bonhage, University of Freiburg, Germany

Amir Omidvarnia, The Florey Institute of Neuroscience and Mental Health, Australia

\section{${ }^{*}$ Correspondence:}

John R. Terry, College of Engineering, Mathematics and Physical Sciences, University of Exeter, Exeter, Devon EX4 4QF, UK

e-mail: j.terry@exeter.ac.uk

${ }^{\dagger}$ Denotes an equal contribution as first author.
Recent clinical work has implicated network structure as critically important in the initiation of seizures in people with idiopathic generalized epilepsies. In line with this idea, functional networks derived from the electroencephalogram (EEG) at rest have been shown to be significantly different in people with generalized epilepsy compared to controls. In particular, the mean node degree of networks from the epilepsy cohort was found to be statistically significantly higher than those of controls. However, the mechanisms by which these network differences can support recurrent transitions into seizures remain unclear. In this study, we use a computational model of the transition into seizure dynamics to explore the dynamic consequences of these differences in functional networks. We demonstrate that networks with higher mean node degree are more prone to generating seizure dynamics in the model and therefore suggest a mechanism by which increased mean node degree of brain networks can cause heightened ictogenicity.

Keywords: network dynamics, epilepsy, dynamical systems, graph theory, EEG

\section{INTRODUCTION}

Epilepsy is a serious neurological disorder characterized by the propensity of the brain to generate spontaneous and recurrent seizures. Traditionally, seizures have been defined as "a transient occurrence of signs and/or symptoms due to abnormal, excessive, or synchronous neural activity in the brain" (1). Very recently, the international league against epilepsy (ILAE) has further refined the definition of epilepsy (2) whereby an individual is now proposed to have epilepsy if one of the following conditions is met:

1. Experiencing two unprovoked seizures more than $24 \mathrm{~h}$ apart.

2. Experiencing a single unprovoked (or reflex) seizure with a probability of further seizures similar to the general risk of recurrence $(\sim 60 \%)$ if two unprovoked seizures had occurred.

3 . An epilepsy syndrome is diagnosed.

It is important to note that epilepsy is a general term to capture over forty, often diverse, syndromes. However, in each case, the generation of clinical signs and symptoms are presumed to require large regions of the brain to be subject to abnormal dynamics and the initiation, recruitment, and spreading of such dynamics is facilitated by the network of synaptic connections between neurons and between regions of the brain. This is reflected in the recognition of the ILAE that many epilepsy syndromes are associated with disruptions to either global or local brain networks (3).

However, a precise definition of global and local brain networks is surprisingly non-trivial. In the global case, one can consider large-scale structural networks as defined by white matter tracts of axons that connect distal brain regions. These networks can be estimated non-invasively using diffusion imaging. An alternative is to examine the statistical inter-relationship between time series recorded at different locations in the brain, thus, defining a "functional" rather than a structural network. While to some extent, functional networks are constrained by the structural architecture of the brain, they also carry contributions from the dynamics of brain activity (4). We recently studied functional networks derived from scalp electroencephalogram (EEG) at rest and demonstrated significant differences between functional networks of people with idiopathic generalized epilepsy (IGE), their first-degree relatives, and healthy controls (5). Significant differences across a number of graph theory measures highlighted abnormalities in both the epilepsy cohort and their first-degree relatives. The most significant of these was that the mean node degree of networks inferred from both people with IGE and their relatives was much greater than that of controls, but that no differences were found between patients and their relatives. This observation suggests that differences between patients and controls cannot be attributed to medication, and thus, altered functional networks are associated with a propensity to generate recurrent seizures (i.e., epilepsy). However, abnormalities in these networks alone are not sufficient to generate seizures (since they are present in the relatives of people with IGE, whom themselves are seizures free) suggesting that the interplay between functional network structure and the dynamics supported by them must play an important role in seizure generating capability (ictogenicity).

The use of mathematical modeling to attempt to address this and related questions has grown substantially in the past few years. Particularly at the macroscopic scale, where the average 
response of a mass of neurons is represented by systems of differential equations, several studies have derived insight into the potential dynamic mechanisms that enable seizures associated with spike-wave discharges to emerge spontaneously from background activity $(6-10)$. Lopes da Silva et al. (11) proposed a scenario in which the spontaneous transitions between background activity and seizure states arise due to bistability, i.e., that the background state and seizure state "coexist" and random inputs can perturb the brain from one state to another. This can be interpreted in terms of either state being able to be reached without a change in underlying constants or slowly varying parameters of the system. This type of model was used to demonstrate that the emergence of either focal or generalized seizure like events could occur due to either specific network disruptions or to alterations in excitability within apparently normal network structures (12).

Motivated by a desire to understand the fundamental mechanisms of seizure transitions more clearly, the concept of bistability has formed the basis of more abstract models of the brain, for example, the so-called $Z^{6}$ model (13), which provides a phenomenological representation of the critical features of more realistic physiological models. These abstract models, which we might consider to represent a normal form of the more detailed physiological representations, have recently been extended to study the role that explicit network structures have in facilitating transitions into seizure activity $(14,15)$.

Here, we build on this previous modeling work to further understand the role of network topology in the generation of transitions into seizure dynamics. In order to understand the potential consequence on ictogenecity of the differences in network structure highlighted by Chowdhury et al. (5), we artificially construct networks that preserve the values of mean node degree for each subject. When these networks are used as the connectivity structure for a bistable dynamic network model, we observe that networks with higher mean node degree transition more readily to a seizure state. We therefore suggest a mechanism by which increased mean node degree of brain networks can cause increased ictogenicity.

\section{MATERIALS AND METHODS MATHEMATICAL MODEL}

Since we focus on the role that network structure plays in transitions between background and seizure states, we do not consider a detailed model of each node in a network. Instead, the foundation of our present work is a network of abstract models that are designed to capture a bistable transition between a "background" state and a high-amplitude "seizure" state [see, e.g., Kalitzin et al. (16)]:

$$
\frac{d}{d t} Z=\left(a|Z|^{4}+b|Z|^{2}+C\right) Z+\varepsilon(t)
$$

where $Z=x+i y$ is a complex variable (function of time); $(a, b)$ are real constant coefficients, and $C=c+i \omega$ is a constant complex coefficient. The term $\varepsilon(t)$ is the complex input to the system, which incorporates a white noise component to mimic the effects of exogenous fluctuations.
A network model, where each node has as its basis the system described in Eq. 1 is then constructed:

$$
\frac{d}{d t} Z_{i}=\left(a\left|Z_{i}\right|^{4}+b\left|Z_{i}\right|^{2}+c+i \omega\right) Z_{i}+\sum_{j=1}^{N} G_{i j} Z_{j}+\varepsilon_{i}(t)
$$

Here, we consider the dynamics of $N$ units, with linear interaction through an adjacency matrix $G$, where white noise is generated independently for each node within the network. In the current work, $G$ is scaled by a factor of 0.1 to preserve transitions between states.

Model parameters are based upon our previous work (16) so that each node lies within the bistable regime. This allows transitions to occur between the steady state (SS), and limit cycle (LC) attractors, where the LC is considered to represent seizure dynamics.

\section{CLINICAL EEG RECORDINGS AND CONSTRUCTION OF FUNCTIONAL NETWORKS}

The network measures that form the basis of this study were inferred from clinical EEG recordings as described in Ref. (5). In brief, these recordings consisted of 19 channel scalp EEG obtained using standard 10-20 placing with an average reference, and sampled at $256 \mathrm{~Hz}$. The recordings were band-passed between 1 and $70 \mathrm{~Hz}$, and notch-filtered between 48 and $52 \mathrm{~Hz}$ to exclude mains frequency interference. The subjects from whom the EEG recordings were taken are divided into two main groups: 35 people with heterogeneous IGE and 40 healthy controls. From each EEG recording, one artifact-free, eyes-closed, $20 \mathrm{~s}$ segment was extracted representing a "resting state" or "background" EEG activity. Chowdhury et al. (5) found significant differences between controls and patients in the 6 and $9 \mathrm{~Hz}$ "low alpha" frequency band, and we therefore focus on that band here. The Hilbert transform was applied to the band-pass filtered EEG to generate instantaneous phase and amplitude estimates. For each electrode pair, the phase-locking factor [PLF, also known as phase-locking value (17) or mean phase coherence (18)] was calculated as follows:

$$
C_{1}=c_{i j}=\frac{1}{N_{s}}\left|\sum_{k=1}^{N_{s}} e^{i \Delta \phi_{i j}\left(t_{k}\right)}\right|
$$

where $\Delta \phi_{i j}\left(t_{k}\right)$ is the instantaneous phase difference between signals $i$ and $j$ at the time point $t_{k}$. The $\Delta \phi_{i j}\left(t_{k}\right)$ were reconstructed from the original signals using the Hilbert transform.

This yields a value between 0 and 1 reflecting the strength of synchronous activity between each pair of signals. Functional networks were then constructed using electrode locations as nodes and PLF values as connectivity weights. Since the PLF measure is symmetrical, the resulting functional connectivity networks are undirected.

\section{NETWORK MEASURES}

The derived functional networks were quantified using the following graph theory measures: mean degree (MD), degree variance (DV), and local clustering coefficient (CC). The degree of a node is defined as the sum of the weights of the edges incident to that 
particular node. The MD and DV are defined as the average and the variance, respectively, of degrees over all nodes in the network. The local CC of a node in a network measures how close its neighbors are to a complete network (graph).

\section{GENERATION OF ARTIFICIAL NETWORKS}

We note that the networks used for connectivity in the model in this study were not directly inferred from patient data, rather "surrogate" networks were prepared, which preserved properties of the networks studied in Chowdhury et al. (5). Each matrix was originally based on the functional connectivity matrix inferred from the aforementioned EEG data. An undirected binary network with the equivalent value of MD as the original matrix was constructed by applying a set of thresholds to the original, and choosing the threshold for which MD was preserved. Further a computational algorithm was applied (19) in order to randomize the matrix, preserving the degree vector and therefore the MD value. In brief, the algorithm randomly swaps nodes and recalculates the degree vector, checking for disparity. For each original matrix, we constructed 30 artificial random binary matrices with the same MD value as the original weighted connectivity matrix. We verified that the spectrum of the artificial patient and control derived networks was different, confirming a difference in topology of the artificial networks.

\section{MEASURE OF BRAIN NETWORK ICTOGENICITY}

We measured the "ictogenicity" of each network by performing simulations using the network as the connectivity matrix for the mathematical model. Since we calculated this measure of ictogenicity from model simulations, we could define an appropriate model state that captured transitions between the non-seizure and seizure dynamics of the model. In the current work, the model seizure state was defined as a solution with local maxima and minima having magnitude $>0.5$.

For each simulation, of the model Eq. 2, we calculated the time that each node spent in a $L C$, normalized to the simulation time. Averaging over all the nodes, we obtain the probability of any node to be in a LC and we refer to this probability as the brain network ictogenicity (BNI).

\section{STATISTICAL ANALYSIS}

For comparison of quantitative network measures between groups, we used a non-parametric Kruskal-Wallis one-way ANOVA test. Results are declared significant for $p<0.05$. For post hoc pairwise comparisons between groups, a Bonferroni corrected multiple comparison test was performed with significance level of 0.05 .

\section{RESULTS}

\section{FUNCTIONAL NETWORKS}

Chowdhury et al. (5) reported that the MD of functional networks derived from people with epilepsy was higher than controls. In Figure 1, we show the distribution of MD for both epilepsy and control subjects included in that study. In this study, we focus on the dynamic consequences of changes in node degree independent of specific network topology and connectivity weights. We remove a layer of complexity from these networks by transforming them into binary (unweighted) networks, while preserving the MD of networks extracted from the EEG data. Figure 1 demonstrates the match in value of mean node degree between the original networks and the artificially derived alternatives (see Materials and Methods).

Since the MD is accurately preserved in our artificial networks, the significant difference in MD between patients and controls is also maintained, as shown in Figure 2. The use of binary, rather than weighted networks leads our artificial networks to have higher DV than the original networks, as demonstrated in Figures 2B,E. A further reason for this difference is that the networks in Chowdhury et al. (5) were normalized to the DV value of 500 surrogate random networks, while in the present case of binary networks such normalization is not possible. However, Figures 2B,E show that a significant difference in DV between epilepsy and control subject derived networks is preserved.

Figures 2C,F demonstrate a lack of significant difference in CC between artificial "control" and "epilepsy" networks, in contrast to the EEG derived networks. This demonstrates that our artificially generated networks have removed some specific topological features of the original data, including those related to clustering.

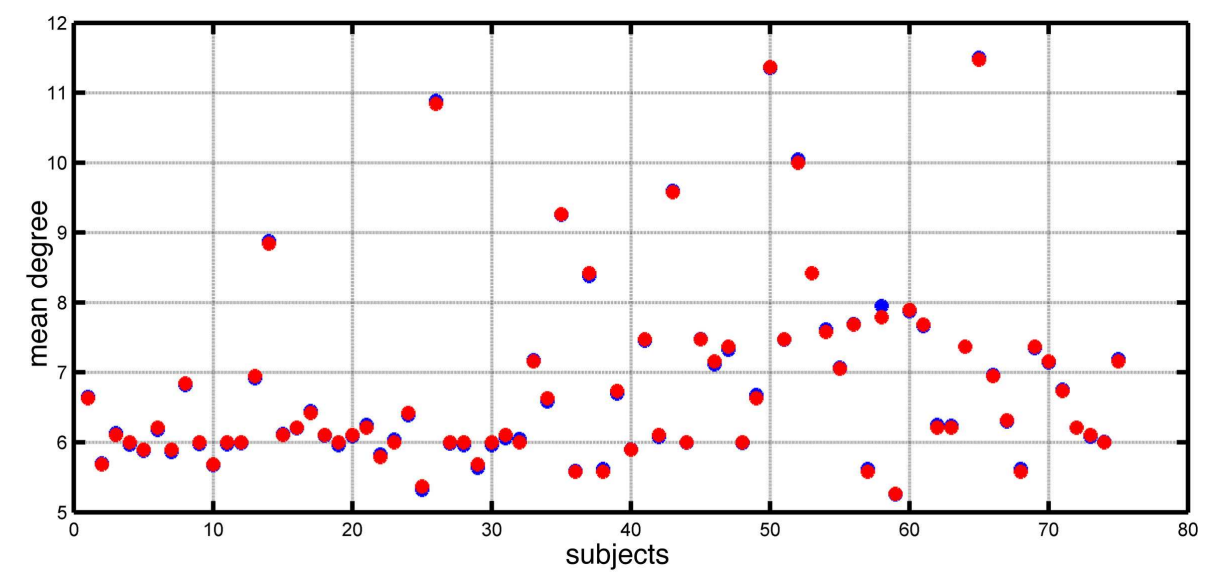

FIGURE 1 | Mean node degree values for each subject (blue dots) and each corresponding artificially constructed binary network (red dots). 


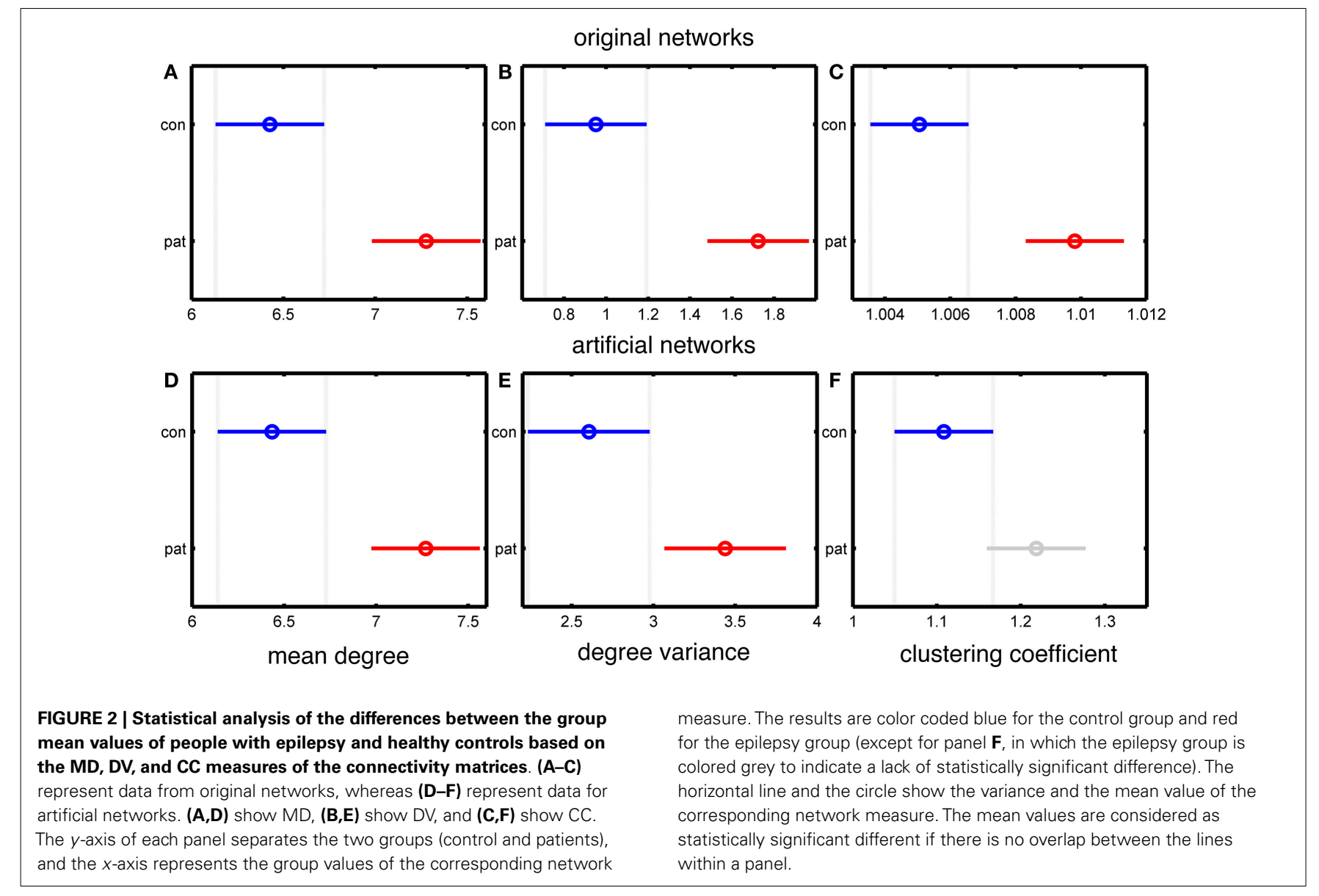

\section{MODELING RESULTS}

For each value of MD extracted from the epilepsy and control cohorts, 30 artificial networks were generated, preserving the MD. These networks were used as the connectivity scheme in the bistable model as described in Section "Materials and Methods." For all simulations of our network model Eq. 2, we fixed model parameters corresponding to the bistable phase space of a single node $\{a, b, c, \omega\}=\{-1,2,-0.9,1+\delta \omega\}$, where $\delta \omega$ is a random number distributed equally in the interval $[-0.2,0.2]$. This choice is made to avoid artificial phase locking because of the equal phase velocities within our multi-unit configuration. For each network, 30 simulations were performed with random initial conditions. The resulting dynamics were quantified according to the BNI measure described in Section "Materials and Methods." An example of the calculation of BNI and the effect of changes in node degree is given in Figure 3.

Four different kinds of dynamics can be seen in Figure 3. In Figure 3A, the model spends a large portion of the simulation time in the "background" attractor before transitioning to the seizure state. Thus, the BNI measurement is low. In contrast, the trajectory of the model in Figures 3B-D moves more quickly into the "seizure" attractor, and so BNI is higher. In addition, in Figure 3C, one of the nodes has not transitioned to the trajectory corresponding to the LC attractor in a single node. Rather, this node is being driven around the corresponding fixed point and therefore the
BNI in this case is lower. It is clear that in this model, BNI provides a measure of how quickly the trajectory of the system performs an "escape" from the background to the seizure attractor.

Figure 4 shows BNI calculated from all simulations for artificial networks derived from the patient and control networks. It can be seen that BNI is significantly higher in the patient versus the control networks, and thus, networks with an increased node degree are shown to have a greater tendency toward seizure activity in this model.

\section{DISCUSSION}

In this study, we used mathematical modeling to investigate the link between the structure of brain networks and their propensity to generate seizure dynamics. Building upon previous studies, we used human EEG data to generate artificial networks preserving MD values, and thus, "isolating" this property for investigation. When networks with high MD were used as connectivity matrices in a model of seizure transitions, we observed significantly more time in the seizure state, as compared to networks with lower MD. We therefore provide evidence for a link between certain properties of network structure (here the MD) and the potential to generate seizure dynamics.

From the network perspective, MD and DV reflect how well connected the nodes within a graph are. Thus, networks with high $\mathrm{MD}$ and low DV would tend toward being fully connected, whereas 


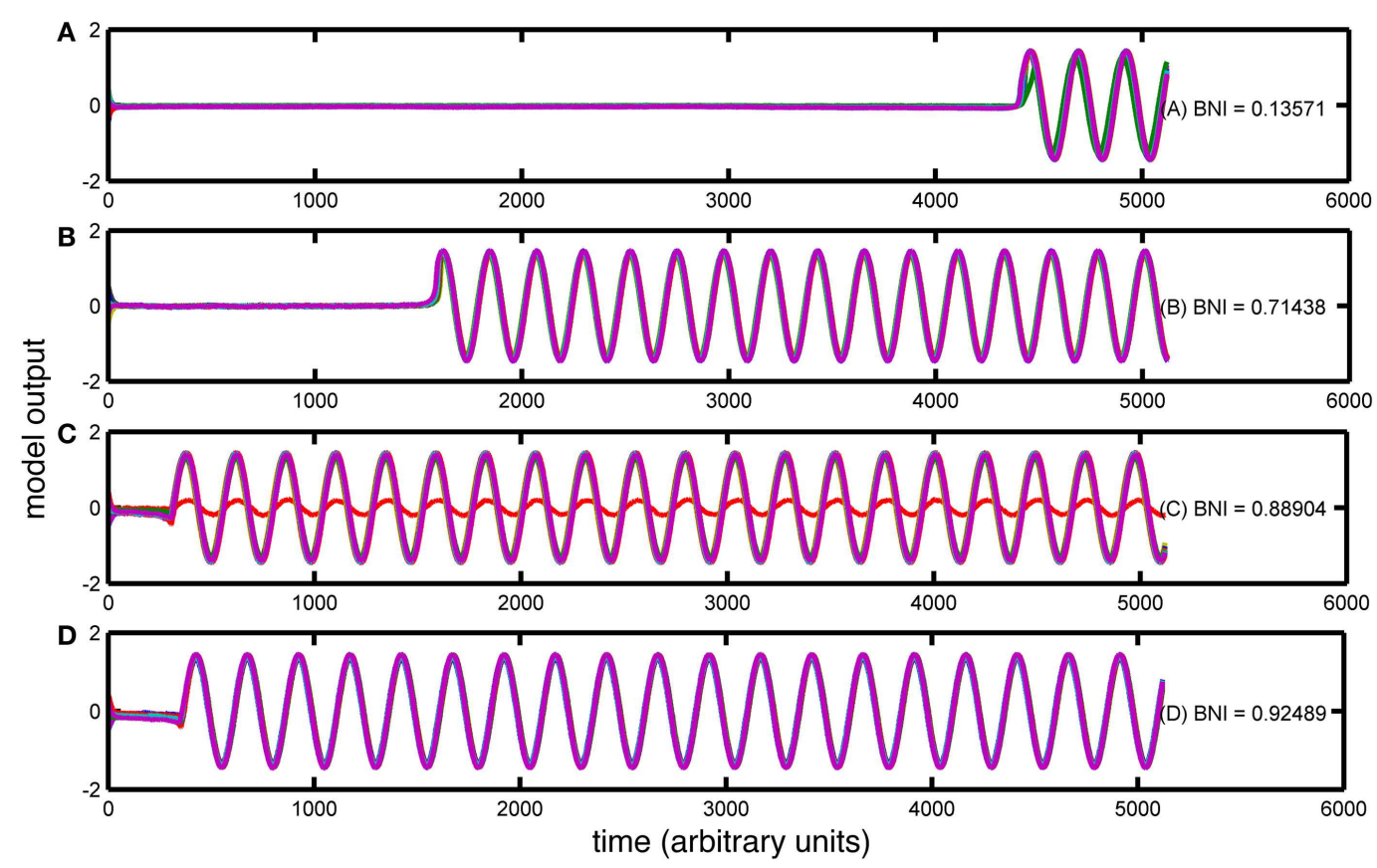

FIGURE 3 | Trajectories of four simulations with the $Z^{6}$ model over different artificially created networks. The figure contains (A-D) four simulations with different BNI values (as indicated). The $x$-axis of each panel represents the simulation time (arbitrary units) and the $y$-axis represents the amplitude of the simulated signal. In each case, all 19 channels are overlaid in different colors. networks with high MD and high DV will have an increased number of "hub-like" nodes. Our randomized networks in the patient group displayed higher MD and DV than controls and therefore fall predominantly into this latter category. This suggests that "hub-like" nodes can more easily drive the rest of the network into the seizure state if they themselves enter that state.

Previous modeling studies in the context of temporal lobe seizures and the hippocampus have suggested a role for hub-like connectivity in generating hyper-excitability (20). Such structures have also been shown to be critical for dementia (21), a condition with which epilepsy is comorbid (22), as well as other pathologies of the brain (23). In a related study, Clemens et al. (24) performed a resting EEG derived, functional connectivity network analysis of people with juvenile myoclonic epilepsy (JME) and control subjects. They found no statistically significant differences in measures of local and global efficiency of the derived networks, where "efficiency" relates to the length of the shortest paths between nodes. We should therefore aim to elucidate exactly which topological features of networks can contribute to the generation of seizure dynamics. In future work, we will explore in more detail the dynamic role of centrality, efficiency, and other features of network topology (25) on seizure generation in our model.

The model employed in this study provides an abstract representation of the epileptic brain. It preserves the potential for transitions between "background" and "seizure" dynamics as postulated in the bistable perspective of generalized seizures (11). This simplified approach allows one to focus upon the role that network structure plays in the propensity for dynamic transitions.
Indeed, this approach has been used with success in terms of estimating transition frequencies (26), exploring the key dynamic components for intermittent transitions (15) and examining the role of specific connection topologies in small networks (14). An interesting extension to the current work would be to assess the interplay between intrinsic node dynamics and network structure. This could be achieved by using abstract models with richer bifurcation structures $(15,27)$, or by employing neural mass models of specific epileptiform dynamics $(8,10,28,29)$.

We built artificial networks preserving MD so as to focus on the implications of changes in this property, with respect to the process of transitions from SS to LC. Precise analysis of the model Eq. 2 leads to the conclusion that the behavior of the system in these terms may depend on several factors such as (a) noise level, (b) initial conditions, (c) connection strength, and (d) network topology. As the main goal was to examine the influence of network topology, we removed the influence of all other factors by setting appropriate noise levels, randomly sampling initial conditions, and using binary instead of weighted networks. In future work, we will consider the effects of adding larger variance noise into the model, in order to facilitate recurrent transitions. In addition, we can expand upon the approach by analyzing weighted networks. We envisage that the addition of these kinds of heterogeneities will lead to a richer repertoire of model dynamics, and therefore, might be useful in further stratifying the effect of network topology on dynamic transitions.

Benjamin et al. (14) examined escape times into seizure dynamics in a similar model applied to networks with a small number of nodes. In that case, it was possible to derive analytic expressions 


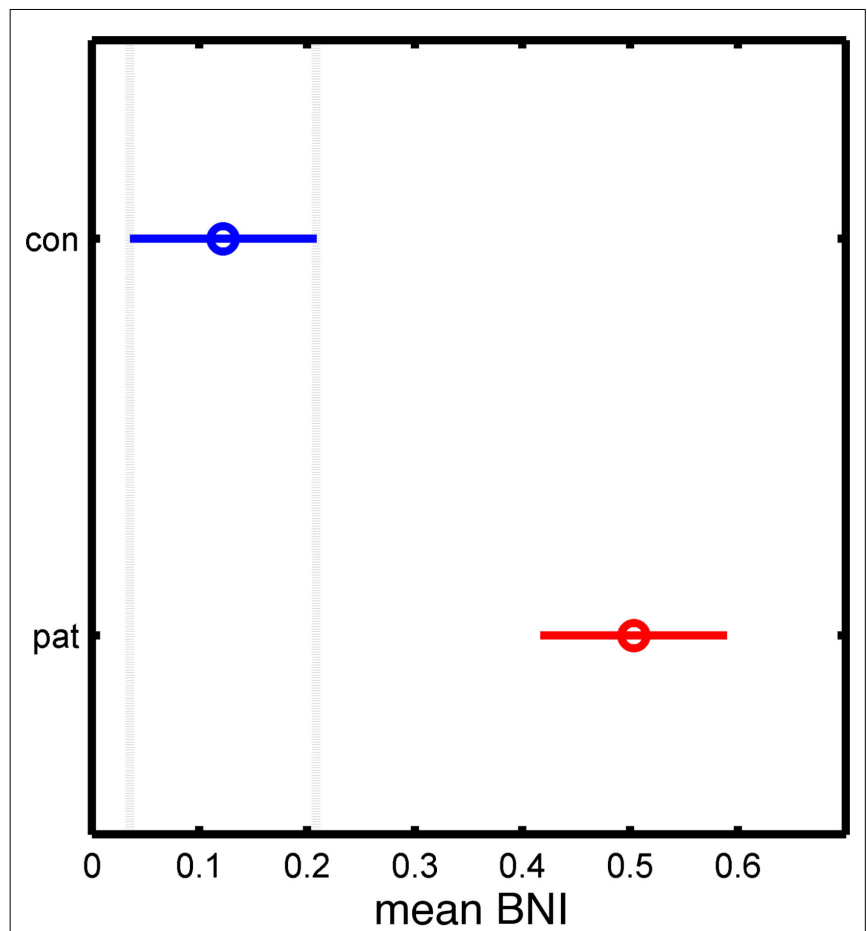

FIGURE 4 | Statistical analysis of the significant differences between control and patient groups based on the mean BNI measure. The $y$-axis separates the two groups, while the $x$-axis represents the mean BNI value. The horizontal line and the circle represent the variance and the mean value of $\mathrm{BNI}$, respectively.

for escape times depending on the topology of networks. However, the complexity of this problem grows significantly as larger networks are considered. Here, in order to link directly with clinical data, we used a model with 19 nodes to represent EEG sensor space. Rather than focusing on explicit network structure, we were able to correlate changes in BNI with properties of the network, e.g., the MD. This provides an avenue to explore the seizure generating potential of more complex networks and could be extended in future work to include other graph theoretic measures, such as the CC, which has also been shown to vary significantly between people with IGE and controls $(5,27)$.

We used functional connectivity as the basis for the networks applied to our model, reflecting the nature of the available clinical data. This approach means that our model is not a direct representation of brain regions interacting over large scales via axonal connections, though such a model can be built in a patient specific way using diffusion data $(29,30)$. Rather, our model provides an abstract representation of the resting state of the brain, as projected onto the level of EEG. Networks derived from this projection are thought to be constrained by structural connectivity, though they are not a direct reflection of it (4). Functional networks by definition represent nodes that are evolving similarly, and therefore, capture a potentially important means by which information can be exchanged between brain regions (31). We should therefore consider that the "connections" of such networks can facilitate the emergence of pathological dynamics through synchronization, and we demonstrate here that this can lead to greater seizure generating potential in the epileptic brain.

On the other hand, functional networks can be viewed more simply as transformations of time series data recorded from subjects, i.e., as mappings from multivariate time series onto a static topological network that reflects a combination of structural and dynamic contributions for that instance of time. From this perspective, our modeling approach gives us a tool with which to interrogate data from people with epilepsy and compare these with control subjects. We therefore aim to explore further whether properties of the BNI derived from functional networks can be used as a marker in the clinical setting. We postulate that in some instances BNI may be able to distinguish between networks that appear similar when examined by traditional graph theoretic measures.

\section{ACKNOWLEDGMENTS}

The authors acknowledge the financial support of the Medical Research Council (MRC) via Programme Grant MR/K013998/01.

\section{REFERENCES}

1. Fisher RS, van Emde Boas W, Blume W, Elger C, Genton P, Lee P, et al. Epileptic seizures and epilepsy: definitions proposed by the international league against epilepsy (ILAE) and the international bureau for epilepsy (IBE). Epilepsia (2005) 46(4):470-2. doi:10.1111/j.0013-9580.2005.66104.x

2. Fisher RS, Acevedo C, Arzimanoglou A, Bogacz A, Cross JH, Elger CE, et al. ILAE official report: a practical clinical definition of epilepsy. Epilepsia (2014) 55(4):475-82. doi:10.1111/epi.12550

3. Berg AT, Berkovic SF, Brodie MJ, Buchhalter J, Cross JH, van Emde Boas W, et al. Revised terminology and concepts for organization of seizures and epilepsies: report of the ILAE commission on classification and terminology, 2005-2009. Epilepsia (2010) 51(4):676-85. doi:10.1111/j.1528-1167.2010.02522.x

4. Honey CJ, Sporns O, Cammoun L, Gigandet X, Thiran JP, Meuli R, et al. Predicting human resting-state functional connectivity from structural connectivity. Proc Natl Acad Sci U S A (2009) 106(6):2035-40. doi:10.1073/pnas.0811168106

5. Chowdhury FA, Woldman W, FitzGerald TH, Elwes RD, Nashef L, Terry JR, et al. Revealing a brain network endophenotype in families with idiopathic generalised epilepsy. PLoS One (2014) 9(10):e110136. doi:10.1371/journal.pone. 0110136

6. Breakspear M, Roberts JA, Terry JR, Rodrigues S, Mahant N, Robinson PA. A unifying explanation of primary generalized seizures through nonlinear brain modeling and bifurcation analysis. Cereb Cortex (2006) 16(9):1296-313. doi:10.1093/cercor/bhj072

7. Suffczynski P, Kalitzin S, Lopes da Silva FH. Dynamics of non-convulsive epileptic phenomena modeled by a bistable neuronal network. Neuroscience (2004) 126(2):467-84. doi:10.1016/j.neuroscience.2004.03.014

8. Goodfellow M, Schindler K, Baier G. Intermittent spike-wave dynamics in a heterogeneous, spatially extended neural mass model. Neuroimage (2011) 55(3):920-32. doi:10.1016/j.neuroimage.2010.12.074

9. Marten F, Rodrigues S, Benjamin O, Richardson MP, Terry JR. Onset of polyspike complexes in a mean-field model of human electroencephalography and its application to absence epilepsy. Philos Trans A Math Phys Eng Sci (2009) 367(1891):1145-61. doi:10.1098/rsta.2008.0255

10. Blenkinsop A, Valentin A, Richardson MP, Terry JR. The dynamic evolution of focal-onset epilepsies - combining theoretical and clinical observations. Eur J Neurosci (2012) 36(2):2188-200. doi:10.1111/j.1460-9568.2012.08082.x

11. Lopes da Silva FH, Blanes W, Kalitzin SN, Parra J, Suffczynski P, Velis DN. Dynamical diseases of brain systems: different routes to epileptic seizures. IEEE Trans Biomed Eng (2003) 50(5):540-8. doi:10.1109/TBME.2003.810703

12. Terry JR, Benjamin O, Richardson MP. Seizure generation: the role of nodes and networks. Epilepsia (2012) 53(9):e166-9. doi:10.1111/j.1528-1167.2012. 03560.x

13. Kalitzin S, Koppert M, Petkov G, Velis D, da Silva FL. Computational model prospective on the observation of proictal states in epileptic neuronal systems. Epilepsy Behav (2011) 22(Suppl 1):S102-9. doi:10.1016/j.yebeh.2011.08.017 
14. Benjamin O, Fitzgerald TH, Ashwin P, Tsaneva-Atanasova K, Chowdhury F, Richardson MP, et al. A phenomenological model of seizure initiation suggests network structure may explain seizure frequency in idiopathic generalised epilepsy. J Math Neurosci (2012) 2(1):1. doi:10.1186/2190-8567-2-1

15. Goodfellow M, Glendinning P. Mechanisms of intermittent state transitions in a coupled heterogeneous oscillator model of epilepsy. J Math Neurosci (2013) 3(1):17. doi:10.1186/2190-8567-3-17

16. Kalitzin S, Koppert M, Petkov G, da Silva FL. Multiple oscillatory states in models of collective neuronal dynamics. Int J Neural Syst (2014) 24(6):1450020. doi:10.1142/S0129065714500208

17. Lachaux JP, Rodriguez E, Martinerie J, Varela FJ. Measuring phase synchrony in brain signals. Hum Brain Mapp (1999) 8(4):194-208. doi:10.1002/(SICI)10970193(1999)8:4<194::AID-HBM4>3.0.CO;2-C

18. Mormann F, Lehnertz K, David P, Elger CE. Mean phase coherence as a measure for phase synchronization and its application to the EEG of epilepsy patients. Physica D (2000) 144(3-4):358-69. doi:10.1016/S0167-2789(00)00087-7

19. Maslov S, Sneppen K. Specificity and stability in topology of protein networks. Science (2002) 296(5569):910-3. doi:10.1126/science.1065103

20. Morgan RJ, Soltesz I. Nonrandom connectivity of the epileptic dentate gyrus predicts a major role for neuronal hubs in seizures. Proc Natl Acad Sci U S A (2008) 105(16):6179-84. doi:10.1073/pnas.0801372105

21. de Haan W, Mott K, van Straaten EC, Scheltens P, Stam CJ. Activity dependent degeneration explains hub vulnerability in Alzheimer's disease. PLoS Comput Biol (2012) 8(8):e1002582. doi:10.1371/journal.pcbi.1002582

22. Noebels J. A perfect storm: converging paths of epilepsy and Alzheimer's dementia intersect in the hippocampal formation. Epilepsia (2011) 52(Suppl 1):39-46. doi:10.1111/j.1528-1167.2010.02909.x

23. Guye M, Bettus G, Bartolomei F, Cozzone PJ. Graph theoretical analysis of structural and functional connectivity MRI in normal and pathological brain networks. MAGMA (2010) 23(5-6):409-21. doi:10.1007/s10334-0100205-z

24. Clemens B, Puskas S, Besenyei M, Spisak T, Opposits G, Hollody K, et al. Neurophysiology of juvenile myoclonic epilepsy: EEG-based network and graph analysis of the interictal and immediate preictal states. Epilepsy Res (2013) 106(3):357-69. doi:10.1016/j.eplepsyres.2013.06.017

25. Strogatz SH. Exploring complex networks. Nature (2001) 410(6825):268-76. doi: $10.1038 / 35065725$
26. Suffczynski P, Lopes da Silva FH, Parra J, Velis DN, Bouwman BM, van Rijn CM, et al. Dynamics of epileptic phenomena determined from statistics of ictaltransitions. IEEE Trans Biomed Eng (2006) 53(3):524-32. doi:10.1109/TBME.2005. 869800

27. Schmidt H, Petkov G, Richardson MP, Terry JR. Dynamics on networks: the role of local dynamics and global networks on the emergence of hypersynchronous neural activity. PLoS Comput Biol (2014) 10(11):e1003947. doi:10.1371/journal. pcbi. 1003947

28. Nevado-Holgado AJ, Marten F, Richardson MP, Terry JR. Characterising the dynamics of EEG waveforms as the path through parameter space of a neural mass model: application to epilepsy seizure evolution. Neuroimage (2012) 59(3):2374-92. doi:10.1016/j.neuroimage.2011.08.111

29. Jirsa VK, Stacey WC, Quilichini PP, Ivanov AI, Bernard C. On the nature of seizure dynamics. Brain (2014) 137(Pt 8):2210-30. doi:10.1093/brain/awu133

30. Ritter P, Schirner M, McIntosh AR, Jirsa VK. The virtual brain integrates computational modeling and multimodal neuroimaging. Brain Connect (2013) 3(2):121-45. doi:10.1089/brain.2012.0120

31. Engel AK, Fries P, Singer W. Dynamic predictions: oscillations and synchrony in top-down processing. Nat Rev Neurosci (2001) 2(10):704-16. doi:10.1038/ 35094565

Conflict of Interest Statement: The authors declare that the research was conducted in the absence of any commercial or financial relationships that could be construed as a potential conflict of interest.

Received: 30 June 2014; accepted: 23 November 2014; published online: 08 December 2014.

Citation: Petkov G, Goodfellow M, Richardson MP and Terry JR (2014) A critical role for network structure in seizure onset: a computational modeling approach. Front. Neurol. 5:261. doi: 10.3389/fneur.2014.00261

This article was submitted to Epilepsy, a section of the journal Frontiers in Neurology. Copyright (C) 2014 Petkov, Goodfellow, Richardson and Terry. This is an open-access article distributed under the terms of the Creative Commons Attribution License (CC $B Y)$. The use, distribution or reproduction in other forums is permitted, provided the original author(s) or licensor are credited and that the original publication in this journal is cited, in accordance with accepted academic practice. No use, distribution or reproduction is permitted which does not comply with these terms. 